\title{
Histerectomía laparoscópica total: manejo ambulatorio. Experiencia Clínica del Prado, Medellín, Colombia
}

\author{
Gustavo Calle G. ${ }^{1}$, José F. De Los Ríos P. ${ }^{1}$, Juan D. Castañeda . $^{1}$, Eduardo \\ Serna A. 1, Ricardo A. Vásquez R. ${ }^{1}$, Adriana M. Arango M. ${ }^{1}$, Henry Muñoz S. ${ }^{2}$, \\ Álvaro J. Escobar F.2, Ana M. Mejía B.a, Germán García S.2 \\ 1 Unidad de Endoscopia Ginecológica, Clínica del Prado, Universidad CES; ${ }^{2}$ Programa en Endoscopia Ginecológica, \\ Universidad CES, Medellín, Colombia.
}

a Instrumentadora Quirúrgica, Unidad de Endoscopia Ginecológica, Clínica del Prado, Universidad de Antioquia, Medellín, Colombia.

\section{RESUMEN}

Objetivo: Describir los resultados de la histerectomía laparoscópica total ambulatoria. Método: Se analizan 297 pacientes sometidas a histerectomía laparoscópica total y que fueron dadas de alta luego de la recuperación anestésica, entre mayo de 2007 y marzo de 2008. Se requería una puntuación de menos de 5 en la escala visual análoga del dolor para el alta. Resultados: El promedio de edad fue de 42 años, el tiempo quirúrgico de 79,1 minutos, el sangrado estimado fue de $37,1 \mathrm{ml}$, el tiempo promedio de estancia total fue de 9,4 horas con un tiempo promedio de estancia postoperatoria de 5,4 horas. La tasa de complicaciones postoperatorias fue de $11,8 \%$ y la de readmisiones del 3,3\%. El promedio de Escala Visual Análoga del dolor para la primera noche fue de 4,2 puntos. El $6 \%$ de las pacientes presentaron vómitos en su domicilio. El 99\% de las pacientes recomendarían este tipo de manejo. Conclusiones: El manejo ambulatorio después de histerectomía laparoscópica total es posible, seguro y bien evaluado por las pacientes. Este manejo no conlleva un mayor riesgo de complicaciones y la tasa de readmisiones es baja.

\section{PALABRAS CLAVE: Histerectomía laparoscópica ambulatoria, complicaciones quirúrgicas, satisfacción de usuaria}

\section{SUMMARY}

Objective: To describe the results of a group of patients in which total laparoscopic hysterectomy was done as an outpatient procedure. Methods: We included 297 patients who were undergone to laparoscopic total hysterectomy, and were discharge to their home after recovery of anesthesia from may 2007 to march 2008. It was necessary to have a punctuation less than 5 in the analogue visual scale to be sent at home. Results: Mean age was 42 years, surgical time 79.1 minutes, estimated blood loss $37.1 \mathrm{ml}$, overall length of stay 9.4 hours, postoperative length of stay 5.4 hours. Postoperative complication rate was $11.8 \%$, readmission rate was $3.3 \%$. Mean VAS Score for pain during the first night was 4.2 points; $6 \%$ of patients vomited at home. Mean VAS Score for satisfaction with ambulatory management was 9.5 , and $99 \%$ of the patients would recommend this type of management. Conclusions: Ambulatory management after total laparoscopic hysterectomy is possible, safe and well evaluated by patients. It does not impose a higher risk of complications and readmission rate is low.

KEY WORDS: Outpatient laparoscopic hysterectomy, surgical complication, user satisfaction 


\section{INTRODUCCIÓN}

Desde su introducción a la práctica clínica, los procedimientos laparoscópicos han contado entre sus ventajas competitivas, con el hecho de disminuir la estancia hospitalaria. Los estudios aleatorizados evaluados por el grupo Cochrane han demostrado consistentemente un menor tiempo de hospitalización al comparar la histerectomía laparoscópica con la vía abdominal, con una diferencia promedio de 3,2 días a favor de la vía laparoscópica (1).

Hace 15 años, Taylor (2), propuso por primera vez el manejo ambulatorio en pacientes llevadas a histerectomía vaginal asistida por laparoscopia. Thiel y Gamelin en 2003 (3), describieron los resultados de 66 pacientes en quienes se practicó histerectomía laparoscópica total y fueron dadas de alta 5 horas después de culminado el procedimiento. Morrison y Jacobs (4), un año más tarde presentan un trabajo con 52 pacientes que estuvieron en una unidad de cirugía ambulatoria, en promedio 6 horas después de histerectomía laparoscópica supracervical. Lieng y cols (5), en 2005 publican su experiencia con 43 mujeres en quienes se realizó histerectomía laparoscópica supracervical con estancia hospitalaria promedio de 5,8 horas después de operadas.

El tiempo de hospitalización necesario para cada procedimiento, más que a una directriz constante, debe obedecer al análisis sensato de los requerimientos de cada paciente, y aunque generalmente se adoptan los rangos necesarios de acuerdo a las costumbres del medio o a las presiones del mismo, debe llevarse a cabo en cada caso particular un análisis que genere una decisión que diste equivalentemente entre una atención de pobre calidad y un exceso de cuidados (6).

Los manejos ambulatorios permiten la recuperación de los pacientes en el ambiente familiar donde se sienten cómodos, disminuyen el riesgo de infección nosocomial (3), y alejan la posibilidad de la comisión de un error médico o de enfermería durante la hospitalización.

En nuestro medio, donde de acuerdo a datos locales no publicados, el tiempo de hospitalización para las histerectomías abdominales o vaginales no va más allá de un día en promedio, hace que la única manera de hacer evidente la ventaja de la vía laparoscópica respecto a este ítem, es intentando replicar los resultados reportados por los autores mencionados empleando un esquema de manejo ambulatorio.

Los análisis de los anteriores factores nos llevaron a instaurar en la Unidad de Endoscopia Ginecológica de la Clínica del Prado, un protocolo de manejo ambulatorio progresivo que debido a sus buenos resultados, se convirtió en la práctica clínica usual para más del $90 \%$ de las pacientes, a quienes se les practica histerectomía laparoscópica total.

El objetivo de este trabajo es presentar nuestra experiencia observada en una cohorte de pacientes sometidas a histerectomía laparoscópica total ambulatoria.

\section{PACIENTES Y MÉTODO}

La muestra consistió en 297 pacientes consecutivas a quienes se les realizó histerectomía laparoscópica total y fueron dadas de alta tras su recuperación anestésica, en la unidad de endoscopia ginecológica de la Clínica del Prado en Medellín, Colombia, entre mayo del 2007 y marzo del 2008. Se incluyeron pacientes con los siguientes criterios: 1. Cirugía sin complicación intraoperatoria evidente. 2. Pacientes con entorno familiar favorable para el manejo ambulatorio (mínimo una persona responsable que viviera con ella). 3. Pacientes cuyo tiempo máximo estimado de acceso a la clínica no fuera mayor de una hora. 4. Que hubieran tolerado la vía oral durante su estancia en el servicio de recuperación. 5. Que hubiesen tenido micción espontánea y normal después del acto quirúrgico. 6 . Sin contraindicaciones para la ingesta de analgésicos y anti-inflamatorios orales. 7. Puntuación de dolor en la escala visual análoga inferior a 5 puntos al momento del alta. Las pacientes que no cumplieron dichos criterios durante el período del estudio no fueron incluidas y fueron manejadas de manera intrahospitalaria.

Los datos acerca de los resultados intra y pos operatorios de esta cohorte de pacientes fueron recopilados en la historia clínica. El tiempo total de estancia hospitalaria se calculó restando a la hora del alta la hora en que la paciente ingresó a la clínica. El tiempo de estancia postoperatoria se calculó restando la hora del alta a la hora en la que la paciente ingresó al servicio de recuperación, según lo registrado en cada historia clínica. Se aplicó una escala visual análoga durante la primera noche del postoperatorio y durante la consulta de revisión entre 7 y 12 días después de la cirugía se aplicó una encuesta de satisfacción y síntomas en el postoperatorio.

Las variables principales en evaluación correspondieron a datos demográficos, tiempo quirúrgico, sangrado estimado, peso uterino, tiempo total de estancia (TTE), tiempo postoperatorio de estancia (TPE), tasa de complicaciones postoperatorias, tasa de readmisión hospitalaria, dolor postoperatorio, tasa de vómito y náuseas y satisfacción. Los 
datos fueron procesados con el software SPSS 15. Este estudio no incurrió en violaciones a la ética. La información fue tomada directamente de la historia clínica teniendo reserva de la identidad de las pacientes, de acuerdo a la declaración de Helsinki y la ley 23 de 1981.

\section{RESULTADOS}

Fueron analizadas un total de 297 pacientes (Tabla I). El promedio de edad de las pacientes fue de 42,7 $\pm 6,03$ años. El tiempo quirúrgico fue de $79,1 \pm 20,9$ minutos (rango: $30-223$ minutos). El sangrado estimado promedio fue de $37,1 \pm 36,3 \mathrm{cc}$ (rango: 5-300 cc). El peso del útero extraído en promedio fue de 196,6 \pm 74.5 gramos (rango: $40-500$ gramos).

\section{Tabla I}

\section{DESCRIPCIÓN DE VARIABLES EVALUADAS}

\begin{tabular}{|c|c|c|}
\hline Variable & Promedio $\pm \mathrm{DE}$ & Rango \\
\hline $\begin{array}{l}\text { Tiempo } \\
\text { quirúrgico (min) }\end{array}$ & $79,1 \pm 20,9$ & $30-223$ \\
\hline $\begin{array}{l}\text { Sangrado } \\
\text { estimado (ml) }\end{array}$ & $37,1 \pm 36,3$ & $5-300$ \\
\hline Peso uterino $(\mathrm{g})$ & $196,6 \pm 74,5$ & $40-500$ \\
\hline $\begin{array}{l}\text { Dosis de } \\
\text { analgésico (número) }\end{array}$ & $2,8 \pm 1,1$ & $1-9$ \\
\hline $\begin{array}{l}\text { Tiempo total } \\
\text { de estancia (horas) }\end{array}$ & $9,41 \pm 1,79$ & $5-12$ \\
\hline $\begin{array}{l}\text { Tiempo posquirúrgico } \\
\text { de estancia (horas) }\end{array}$ & $5,38+/-1,8$ & $2-9$ \\
\hline
\end{tabular}

EI TTE en promedio fue de $9,4 \pm 1,8$ horas ( 5 - $12 \mathrm{~h}$ ) mientras que el TPE fue de 5,38 $\pm 1,8$ horas (rango: 2-9 horas). La tasa de complicaciones postoperatorias fue de $11,8 \%$ de las cuales 15 pacientes $(5 \%)$ presentaron infección de cúpula vaginal, 10 pacientes $(3,3 \%)$ tuvieron hematoma de cúpula, $5(1,6 \%)$ presentaron sangrado tardío de cúpula, $4(1,6 \%)$ infección de vías urinarias y hubo 1 caso $(0,3 \%)$ de dehiscencia de cúpula.

En las primeras 48 horas se presentaron 10 readmisiones entre las 297 pacientes $(3,3 \%)$, las cuales 5 fueron por fiebre subjetiva y 5 por dolor. El manejo ambulatorio del dolor se manejo con ibuprofeno $600 \mathrm{mg}$ cada 6 horas y acetaminofen 500 mg cada 6 horas. El promedio de la Escala Visual Análoga (VAS) del dolor durante la primera noche fue de $4,17 \pm 2,88$. El $47,5 \%$ de las pacientes experimentaron dolor mínimo y $7 \%$ necesitaron diferentes esquemas de analgesia con diclofenaco y tramadol. El $6 \%$ de las pacientes presentaron vomito en casa un promedio de 3,2 veces.

La satisfacción respecto al manejo ambulatorio fue de $9,5 \pm 1,26$ (escala de 0 a 10). Un $92 \%$ de las pacientes se sintieron confortables con el manejo ambulatorio y un $99 \%$ de las pacientes recomendarían este tipo de manejo a un miembro de su familia.

\section{DISCUSIÓN}

Una de las ventajas importantes de la cirugía mínimamente invasiva es su pronta recuperación comparada con la cirugía abierta y la posibilidad de manejar a las pacientes en una forma ambulatoria. La histerectomía no es la excepción y los resultados de nuestra experiencia con la histerectomía laparoscópica total muestran un nivel alto de satisfacción de las usuarias y una tasa de complicaciones similar a la reportada para este procedimiento en diferentes publicaciones $(1,7,8)$.

Respecto a la estancia hospitalaria encontramos un promedio inferior al reportado en la mayoría de las publicaciones y similar al reportado por Thiel y Kamensic (9) en su experiencia con un grupo de 224 pacientes.

La histerectomía laparoscópica ha ganado popularidad por los beneficios que ha demostrado sobre la histerectomía por laparotomía. En 1999, Chapron y cols (10), mostraron que sólo el 13,2\% de las pacientes se operaban por esta vía, lo cual se explica por el mayor entrenamiento quirúrgico que se requiere. Se destacan sus mejores resultados cosméticos y menores complicaciones infecciosas y de la pared abdominal.

En 1999 Chou y cols (11), presentaron una serie donde demuestra que en $90 \%$ de los casos de histerectomía laparoscópica, las pacientes fueron dadas de alta en las primeras 24 horas y resalta la reducción de los costos de la estancia hospitalaria y la alta satisfacción de las pacientes con esta técnica. En su discusión plantea como de esta forma se liberan camas y se acortan las listas de espera para cirugía que anteriormente requería hospitalizaciones prolongadas.

Moller y cols (12) en una serie de 32 pacientes sometidas a histerectomía, 16 por vía abdominal y 16 por vía vaginal asistida por laparoscopia, reportan mayor tiempo de cirugía y de anestesia para las pacientes de la vía laparoscópica, y no encuentra diferencias en el tiempo fuera de la cama 
en la primera semana postoperatoria; además las por vía abdominal demoran más en reintegrarse al trabajo pese a que la estancia hospitalaria promedio fue de 1 día para la vía laparoscópica y de 2 para la vía abierta.

En la evaluación de la seguridad y la satisfacción de las pacientes sometidas a histerectomía por laparoscopia y manejo ambulatorio, Thiel y Gamelin (3), reportan que el $92,5 \%$ de las 66 pacientes de su serie fueron dadas de alta el mismo día y que el 95\% de las pacientes lo recomendarían, lo cual da cuenta de que es un procedimiento bien tolerado. En ese artículo, reconocido por los autores como el primero que informa sobre el tratamiento ambulatorio de pacientes sometidas a histerectomía por laparoscopia, la incidencia de complicaciones fue del $16 \%$ y correspondió a situaciones menores que no revistieron mayor importancia, como la necesidad de antibióticos o el drenaje de un hematoma de la cúpula.

Más recientemente Candiani y cols (13), presenta los datos de un estudio prospectivo en el que se comparó la histerectomía laparoscópica contra la vaginal. Aunque la duración de la cirugía fue estadísticamente mayor en la vía laparoscópica, hubo diferencias significativas a favor de la laparoscopia en relación con el sangrado y la estancia hospitalaria. Sobre estos resultados se cuestionan si la vía vaginal debe ser considerada el estándar general y particularmente en los casos donde esté indicada la anexectomía, que es más factible por laparoscopia que por la vía vaginal.

Una de las ventajas importantes de la cirugía mínimamente invasiva es su pronta recuperación comparada con la cirugía abierta y la posibilidad de manejar a las pacientes ambulatoriamente. La histerectomía no es la excepción y los resultados de nuestra experiencia con la histerectomía laparoscópica total muestran un nivel alto de satisfacción por parte de las usuarias y una tasa de complicaciones similar a la reportada para este procedimiento en diferentes publicaciones $(1,7,8)$.

Respecto a la estancia hospitalaria encontramos un promedio inferior al reportado en la mayoría de las publicaciones y similar al reportado por Thiel y cols en su experiencia en 224 pacientes (9).

\section{CONCLUSIÓN}

El manejo ambulatorio después de la histerectomía laparoscópica total es factible, seguro y bien calificado por las pacientes. El manejo ambulatorio no implica un riesgo mayor de complicaciones respecto al manejo hospitalario y presenta una tasa baja de readmisiones.

\section{BIBLIOGRAFÍA}

1. Nieboer TE, Johnson N, Lethaby A, Tavender E, Curr E, Garry R, et al. Surgical approach to hysterectomy for benign gynaecological disease. Cochrane Database of Systematic Reviews 2009, Issue 3. No.: CD003677. DOI: 10.1002/14651858.CD003677.

2. Taylor RH. Outpatient laparoscopic hysterectomy with discharge in 4 to 6 hours. J Am Assoc Gynecol Laparosc 1994;1(4, Part 2):S35.

3. Thiel J, Gamelin A. Outpatient total laparoscopic hysterectomy. J Am Assoc Gynecol Laparosc 2003;10:481-3.

4. Morrison JE Jr, Jacobs VR. Outpatient laparoscopic hysterectomy in a rural ambulatory surgery center. $\mathrm{J}$ Am Assoc Gynecol Laparosc 2004;11:359-64.

5. Lieng $M$, Istre $O$, Langebrekke $A$, Jungersen $M$, Busund $B$. Outpatient laparoscopic supracervical hysterectomy with assistance of the lap loop. J Minim Invasive Gynecol 2005;12:290-4.

6. Hollenbeck BK, Dunn RL, Gilbert SM, Strope S, Miller DC. Effects of laparoscopy on surgical discharge practice patterns. Urology 2008;71:1029-34.

7. Garry R, Fountain J , Mason S, Napp V, Brown J, Hawe J, et al. The eVALuate study: two parallel randomized trials, one comparing laparoscopic with abdominal hysterectomy, the other comparing laparoscopic with vaginal hysterectomy. BMJ 2004;328:129-33.

8. Warren L, MD, Ladapo JA, Borah BJ, Candace L. Gunnarsson CL. Open abdominal versus laparoscopic and vaginal hysterectomy: analysis of a large united states payer measuring quality and cost of care. $\mathrm{J} \mathrm{Mi-}$ nim Invasive Gynecol 2009;16:581-8.

9. Thiel JA, Kamencic H. Assessment of costs associated with outpatient total laparoscopic hysterectomy. J Obstet Gynaecol Can 2006;28:794-8.

10. Chapron C, Laforest L, Ansquer Y, Fauconnier A, Fernandez B, Bréart G, Dubuisson JB. Hysterectomy techniques used for benign pathologies: results of a french multicentre study. Human Reproduction 1999;14:2464-70.

11. Chou DC, Rosen DM, Cario GM, Carlton MA, Lam AM, Chapman M, Johns C. Home within 24 hours of laparoscopic hysterectomy. Aust NZ J Obstet Gymaecol 1999;39:234-8.

12. Moller C, Kehlet H, Friland SG, Schouenborg LO, Lund C, Ottesen B. Fast track hysterectomy. Eur J Obstet Gynecol Reprod Biol 2001;98:18-22.

13. Candiani M, Izzo S, Bulfoni A, Riparini J, Ronzoni S, Marconi A. Laparoscopic vs vaginal hysterectomy for benign pathology. Am J Obstet Gynecol. 2009;200(4):368.e1-7. 